\title{
EFEITO DE ADUBOS POTÁSSICOS NA PRODUÇÃO DE SOJA ${ }^{1}$
}

\author{
H.A.A. MASCARENHAS ${ }^{2}$; R.T. TANAKA ${ }^{2}$ \\ Seção de Leguminosas/lAC, C.P. 28, CEP: 13001-970 Campinas,SP. \\ J.C.V.N.A. PEREIRA \\ Estação Experimental de Ribeirāo Preto/IAC \\ P.B. GALLO \\ Estaçäo Experimental de Mococa/IAC \\ O.C. BATAGLIA ${ }^{2}$ \\ Seção de Fertilidade do Solo e Nutrição de Plantas/IAC, C.P. 28, CEP: 13001-970 Campinas,SP
}

RESUMO: Um experimento de adubos potássicos (KCl, K2SO4, K-Mag e vinhaça) foi conduzido durante três anos agricolas em quatro classes de solo: Podzolico Vermelho Amarelo Orto (PV), Latossolo Roxo distrófico (LR), Latossolo Vermelho Escuro textura média (LE) e Latossolo Vermelho Amarelo textura média (LV). Os três primeiros adubos foram aplicados somente no primeiro cultivo da soja na dose de $150 \mathrm{~kg} / \mathrm{ha}$ de $\mathrm{K}_{2} \mathrm{O}$ a lanço e incorporados, enquanto a vinhaça foi aplicada anualmente na dose de $50.000 \mathrm{l} / \mathrm{ha}$, um pouco antes de semeadura da soja. Os resultados mostraram que os cultivos sucessivos diminúram gradativamente os teores de potássio tanto no solo como nas folhas. No segundo ano de cultivo em LV houve respostas positivas da cultura da soja ao uso de potássio, enquanto em PV, no terceiro ano. Entretanto, não se observaram diferenças entre os adubos. $O$ cultivar IAC-9 mostrou alta capacidade extratora de $\mathrm{K}$ como de $\mathrm{Ca}$ e $\mathrm{Mg}$.

Descritores: soja, cloreto de potássio, sulfato de potássio, K-Mag, vinhaça

\section{EFFECT OF POTASSIUM FERTILIZERS ON SOYBEAN YIELD}

\begin{abstract}
Experiments of potassium fertilizers (KCl, K2SO4, $\mathrm{K}-\mathrm{Mag}$ and vinasse) were conducted in four types of soil: Ortho Red Yellow Podzolic (PV), Dusky distrophic Latosol (LR), Dark Red medium texture Latosol (LE), and Red Yellow medium texture Latosol (LV). The first three fertilizers were applied broadcast at the rate of $150 \mathrm{~kg} / \mathrm{ha}$ of $\mathrm{K}_{2} \mathrm{O}$ in the first year, whereas vinasse was applied annually at the rate of $50,000 \mathrm{l} / \mathrm{ha}$ just before planting soybeans. The results showed that there was a decrease in the concentration of potassium, both in the soil and leaves, anually, during the three years for all four soils. There was a positive response in the yield of soybeans to potassium in the LV soil on the second year and in the PV soil on the third year, however no diferences among potassium fertilizers was observed. The cultivar IAC-9 showed its high capacity to extract not only $\mathrm{K}$ but also $\mathrm{Ca}$ and $\mathrm{Mg}$.
\end{abstract}

Key Words: soybeans, potassium chloride, potassium sulphate, K-Mag, vinasse

\section{INTRODUÇÃO}

Cerca de $95 \%$ do potássio aplicado em culturas é na forma de cloreto de potássio em virtude do seu preço. Por outro lado, o sulfato de potássio, mais caro que o cloreto de potássio (SEARLS, 1983), é recomendado para as culturas do fumo, batata, beterraba e algumas frutíferas. Nos Estados Unidos em solos orgânicos mal drenados de Georgia, PARKER et al. (1983) observaram toxidez de cloro (quando a fonte aplicada de potássio foi cloreto) nas plantas de soja de alguns cultivares e queda na produtividade em um ano de baixa precipitação pluvial. No ano

\footnotetext{
${ }^{1}$ Trabalho realizado com apoio financeiro do Convênio MIC/STI/FUNAT/IAC.

2 Bolsista do CNPq.
} 
seguinte foi necessária a utilização de $\mathrm{K}_{2} \mathrm{SO}_{4}$ como fonte de potássio nestes cultivares de alta produtividade.

Em 1980 foi trazido dos Estados Unidos para o Brasil, o fertilizante K-Mag para ser utilizado como fonte de $\mathrm{K}, \mathrm{Mg}$ e S. Também nesta época a vinhaça ganhou importância como fonte de potássio para culturas extensivas como a cana de açúcar.

O objetivo deste trabalho foi avaliar o efeito dos adubos $\mathrm{KCl}, \mathrm{K} 2 \mathrm{SO} 4, \mathrm{~K}-\mathrm{Mag}$ e da vinhaça na produtividade de soja.

\section{MATERIAL E MÉTODOS}

Os experimentos foram conduzidos durante três anos agrícolas nos municípios de Orlândia (Latossolo Vermelho Escuro textura média), Mococa (Podzólico Vermelho Amarelo Orto), Ribeirão Preto (Latossolo Roxo distrófico), e Paraguaçu Paulista (Latossolo Vermelho Amarelo textura média). As caracteríticas químicas desses solos coletados antes da aplicação de calcário estão apresentados na TABELA 1 . Solos dos ensaios de Mococa e Ribeirão Preto estão em níveis médios de $\mathrm{K}$ e baixos nos de Orlândia e Paraguaçu Paulista. Esses municípios estão inseridos em regiões onde há cultivo da soja perto das usinas de açúcar e álcool que produzem a vinhaça como resíduo. A correção do complexo acidez foi feita com calcário dolomítico com a finalidade de atingir os teores de $\mathrm{Ca}$ mais $\mathrm{Mg}$ trocáveis a $3 \mathrm{meq} / 100 \mathrm{ml}$. $\mathrm{O}$ delineamento experimental foi em blocos ao acaso com cinco repetições.

Os tratamentos consistiram da aplicação a lanço dos seguintes adubos potássicos:

\section{Testemunha.}

2. Cloreto de potássio $\left(60 \% \mathrm{~K}_{2} \mathrm{O}\right.$ e $\left.45-48 \% \mathrm{Cl}\right)$

3. Sulfato de potássio ( $48 \% \mathrm{~K}_{2} \mathrm{O}$ e $15-17 \% \mathrm{~S}$ )

4. K-MAG (22\% $\mathrm{K}_{2} \mathrm{O}, 18 \% \mathrm{MgO}$ e $\left.15-17 \% \mathrm{~S}\right)$

5. Vinhaça

As concentrações de todos os nutrientes da vinhaça foram variáveis entre os anos de aplicação e entre os ensaios conforme mostra a TABELA 2.

Os adubos sólidos foram aplicados somente a lanço e incorporados antes do primeiro cultivo na dose de $150 \mathrm{~kg} / \mathrm{ha}$ de $\mathrm{K}_{2} \mathrm{O}$, enquanto a vinhaça foi aplicada anualmente na dose a $50.0001 /$ ha. A cada ano a adubação aplicada no sulco foi de $60 \mathrm{~kg} / \mathrm{ha}$ de $\mathrm{P}_{2} \mathrm{O}_{5}$ na forma de superfosfato simples.

O cultivar utilizado de soja foi IAC-9 cujas sementes foram tratadas com Thiabendozole (contra doenças fúngicas de sementes) e inoculadas com Bradyrhizobium japonicum para garantir a fixação de nitrogênio atmosférico. As parcelas consistiram de quatro linhas de cinco metros e espaçadas entre si de $0,60 \mathrm{~m}$, em densidade de 20 plantas/metro linear. No início do florescimento foram feitas amostragens das terceiras folhas a partir do ápice das plantas num total de trinta por parcela. As análises de macronutrientes (exceto $0 \mathrm{~S}$ ) foram feitas de acordo com os métodos descritos por BATAGLIA et al. (1983).

A colheita de soja foi feita das duas linhas centrais excluindo $0,50 \mathrm{~m}$ de cada extremidade. Após a colheita, o solo foi amostrado na camada de 0-20 cm de cada parcela, sendo as análises químicas efetuadas segundo os métodos descritos por RAIJ \& QUAGGIO (1983).

\section{RESULTADOS E DISCUSSÃo}

A TABELA 3 apresenta as produtividades de soja do ensaio de Orlândia na qual não se observa diferenças entre os adubos de potássio inclusive com a testemunha, durante os três anos agrícolas. Apenas houve diferenças significativas para anos.

A TABELA 4 mostra os teores de macronutrientes nas folhas de soja. Observa-se que o teor de potássio diminuiu a cada cultivo com todos os tratamentos inclusive a de testemunha. Apesar desta tendência todos os teores estão dentro de limites considerados adequados $(1,71-2,50 \%)$ conforme OHLROGGE \& KAMPRATH (1968). O teor de K disponível do solo no tratamento testemunha, contrariando os dados da TABELA 1, impediu provavelmente que houvesse reposta da soja às fontes de $\mathrm{K}$ (TABELA 3).

Os resultados da análise do solo (TABELA 5) comprovam que os teores de potássio e também de cálcio e magnésio diminuíram com os cultivos, atingindo em $1982 / 83$ o nível muito baixo independente do tratamento.

Apesar desta deficiência no solo, a soja IAC-9 provavelmente pela sua alta capacidade de extrair potássio e magnésio apresentaram seus teores em níveis adequados nas folhas (TABELA 4).

Somente no terceiro cultivo do ensaio de Mococa, houve efeito do $\mathrm{K}$ sobre a produtividade de soja onde o cloreto de potássio e a vinhaça mostraram ser superiores à testemunha (TABELA 6) não tendo havido diferenças entre as fontes.

Como no ensaio de Orlândia, os teores de potássio nas folhas diminuíram com os cultivos e no terceiro, o teor nas folhas da testemunha 
TABELA 1 - Resultados das análises do solo antes da aplicação de calcário.

\begin{tabular}{lcccc}
\hline \multicolumn{1}{c}{ Análise $^{1}$} & Orlândia & Mococa & Ribeirão Preto & Paraguaçu Paulista \\
\hline pH & 5,2 & 5,4 & 5,1 & 5,0 \\
M.o. \% & 1,7 & 2,6 & 3,7 & 1,5 \\
P ug & 1,5 & 5,6 & 3,7 & 1,5 \\
K meq/100 ml & 0,06 & 0,15 & 0,13 & 0,03 \\
Ca meq/100 ml & 0,4 & 2,0 & 2,5 & 0,9 \\
Mg meq/100 ml & 0,3 & 0,4 & 0,6 & 0,5 \\
Al meq/100 ml & 0,6 & 0,8 & 0,5 & 0,3 \\
\hline \hline
\end{tabular}

${ }^{1} \mathrm{pH}$ em água, $\mathrm{P}$ e K em $\mathrm{H}_{2} \mathrm{SO}_{4} 0,05 \mathrm{~N}$ e $\mathrm{Ca}, \mathrm{Mg}$ e $\mathrm{Al} \mathrm{em} \mathrm{KCl} 1 \mathrm{~N}$.

TABELA 2 - Resultados das análises químicas da vinhaça utilizada durante três cultivos e em quatro localidades.

\begin{tabular}{|c|c|c|c|c|c|c|c|c|c|c|c|}
\hline Cultivos & C & $\mathbf{N}$ & $\mathbf{P}$ & $\mathrm{Ca}$ & $\mathrm{Mg}$ & $\mathbf{K}$ & $\mathrm{Na}$ & $\mathrm{Fe}$ & Mn & $\mathrm{Cu}$ & $\mathrm{Zn}$ \\
\hline & $\%$ & $\%$ & $\cdots$ & & & $\cdots$ & ppm . & & & & $\cdots$ \\
\hline & \multicolumn{11}{|c|}{ ORLÂNDIA } \\
\hline $1980 / 81$ & 1,6 & 0,11 & 109 & 177 & 145 & 1209 & 21 & 181 & 11 & 0,9 & 120 \\
\hline $1981 / 82$ & 1,0 & 0,06 & - & 384 & 195 & 1600 & 37 & 104 & 5 & $<2$ & 68 \\
\hline \multirow[t]{2}{*}{$1982 / 83$} & 1,2 & 0,05 & - & 820 & 180 & 4600 & 28 & 30 & 6 & $<4$ & 9 \\
\hline & \multicolumn{11}{|c|}{ PARAGUAÇU PAULISTA } \\
\hline $1980 / 81$ & 1,8 & 0,11 & 46 & 1610 & 517 & 4173 & 180 & 850 & 15 & 2,7 & 13 \\
\hline $1981 / 82$ & 0,8 & 0,04 & - & 522 & 197 & 1400 & 55 & 49 & 7 & $<2$ & 41 \\
\hline \multirow[t]{2}{*}{$1982 / 83$} & 0,8 & 0,05 & - & 560 & 109 & 2000 & 41 & 26 & 4 & $<4$ & 10 \\
\hline & \multicolumn{11}{|c|}{ RIBEIRÃO PRETO } \\
\hline $1980 / 81$ & 1,5 & 0,05 & 41 & 1329 & 521 & 4056 & 124 & 97 & 12 & 0,5 & 13 \\
\hline $1981 / 82$ & 1,8 & 0,08 & - & 660 & 491 & 3200 & 193 & 281 & 7 & 17 & 4 \\
\hline \multirow[t]{2}{*}{$1982 / 83$} & 1,0 & 0,04 & - & 560 & 180 & 600 & 51 & 500 & 12 & $<4$ & 1 \\
\hline & \multicolumn{11}{|c|}{ MOCOCA } \\
\hline $1980 / 81$ & 0,8 & 0,04 & 17 & 667 & 183 & 1424 & 28 & 216 & 6 & 0,4 & 20 \\
\hline $1981 / 82$ & 0,9 & 0,05 & - & 558 & 176 & 2080 & 55 & 276 & 7 & $<2$ & 168 \\
\hline $1982 / 83$ & 2,1 & 0,16 & - & 600 & 120 & 3200 & - & 67 & 10 & $<4$ & 60 \\
\hline
\end{tabular}


TABELA 3 - Efeito da aplicação de adubos potássicos, na produtividade de soja durante três anos agrícolas no ensaio de Orlândia.

\begin{tabular}{|c|c|c|c|c|}
\hline Tratamentos & $1980 / 81$ & $1981 / 82$ & $1982 / 83$ & Média \\
\hline & \multicolumn{4}{|c|}{ Grãos kg/ha } \\
\hline Testemunha & 2107 & 2504 & 2625 & 2412 \\
\hline $\mathrm{KCl}$ & 2332 & 2454 & 2725 & 2504 \\
\hline $\mathrm{K}_{2} \mathrm{SO}_{4}$ & 1922 & 2525 & 2737 & 2395 \\
\hline K-Mag & 2126 & 2483 & 2848 & 2486 \\
\hline Vinhaça & 2271 & 2381 & 2592 & 2415 \\
\hline Média & 2152 & 2469 & 2705 & 2442 \\
\hline CV \% & 13,8 & 8,3 & 10,7 & 11,0 \\
\hline
\end{tabular}

TABELA 4 - Efeito da aplicação de adubos potássicos nos teores de macronutrientes nas folhas de soja de três anos agrícolas no ensaio de Orlândia.

\begin{tabular}{|c|c|c|c|c|c|c|c|c|c|c|c|c|c|c|c|}
\hline \multirow{2}{*}{$\begin{array}{l}\text { Trata- } \\
\text { mentos }\end{array}$} & \multicolumn{5}{|c|}{$1980 / 81$} & \multicolumn{5}{|c|}{$1981 / 82$} & \multicolumn{5}{|c|}{$1982 / 83$} \\
\hline & $\mathbf{N}$ & $\mathbf{P}$ & $\mathbf{K}$ & $\mathrm{Ca}$ & $\mathbf{M g}$ & $\mathbf{N}$ & $\mathbf{P}$ & $\mathbf{K}$ & $\mathrm{Ca}$ & $\mathbf{M g}$ & $\mathbf{N}$ & $\mathbf{P}$ & $\mathbf{K}$ & $\mathrm{Ca}$ & $\mathbf{M g}$ \\
\hline & & & & & & & & $\%$ & & & & & & ........ & \\
\hline Test. & 4,0 & 0,33 & 2,9 & 1,0 & 0,46 & 3,4 & 0,25 & 2,1 & 1,6 & 0,48 & 4,8 & 0,32 & 2,1 & 1,4 & 0,39 \\
\hline $\mathrm{KCl}$ & 3,5 & 0,33 & 3,1 & 1,0 & 0,41 & 3,9 & 0,24 & 2,4 & 1,5 & 0,41 & 4,4 & 0,32 & 2,1 & 1,4 & 0,41 \\
\hline $\mathrm{K}_{2} \mathrm{SO}_{4}$ & 3,4 & 0,32 & 2,9 & 1,0 & 0,44 & 3,5 & 0,24 & 2,3 & 1,3 & 0,45 & 4,5 & 0,33 & 2,1 & 1,4 & 0,39 \\
\hline K-Mag & 3,2 & 0,34 & 3,1 & 1,0 & 0,47 & 3,7 & 0,27 & 2,7 & 1,3 & 0,49 & 4,5 & 0,33 & 2,1 & 1,4 & 0,39 \\
\hline Vinh. & 3,2 & 0,36 & 3,0 & 1,0 & 0,43 & 4,0 & 0,27 & 2,2 & 1,6 & 0,53 & 4,6 & 0,32 & 2,0 & 1,4 & 0,43 \\
\hline
\end{tabular}

TABELA 5 - Resultados da análise química do solo após a colheita de soja no ensaio de Orlândia.

\begin{tabular}{|c|c|c|c|c|c|c|c|c|c|}
\hline \multirow[b]{2}{*}{ Tratamentos } & \multicolumn{3}{|c|}{$1980 / 81$} & \multicolumn{3}{|c|}{$1981 / 82$} & \multicolumn{3}{|c|}{$1982 / 83$} \\
\hline & $\mathbf{K}$ & $\mathrm{Ca}$ & $\mathbf{M g}$ & K & $\mathrm{Ca}$ & $\mathbf{M g}$ & $\mathbf{K}$ & $\mathrm{Ca}$ & $\mathbf{M g}$ \\
\hline & \multicolumn{9}{|c|}{ 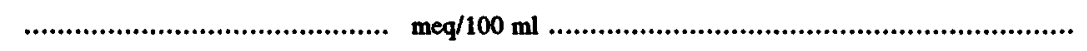 } \\
\hline Testemunha & 0,13 & 1,2 & 0,3 & 0,11 & 0,5 & 0,2 & 0,04 & 1,0 & 0,1 \\
\hline $\mathbf{K C l}$ & 0,18 & 1,3 & 0,3 & 0,13 & 0,7 & 0,2 & 0,04 & 1,1 & 0,2 \\
\hline $\mathrm{K}_{2} \mathrm{SO}_{4}$ & 0,12 & 1,1 & 0,3 & 0,11 & 0,6 & 0,2 & 0,04 & 0,9 & 0,1 \\
\hline K-Mag & 0,20 & 1,3 & 0,4 & 0,17 & 0,8 & 0,2 & 0,05 & 1,5 & 0,2 \\
\hline Vinhaca & 0,17 & 1,3 & 0,3 & 0,10 & 0,6 & 0,2 & 0,05 & 1,1 & 0,1 \\
\hline
\end{tabular}


TABELA 6 - Efeito da aplicação de adubos potássicos na produtividade de soja durante três anos agrícolas no ensaio de Mococa.

\begin{tabular}{|c|c|c|c|c|}
\hline Tratamentos & $1980 / 81$ & $1981 / 82$ & $1982 / 83$ & Média \\
\hline & \multicolumn{4}{|c|}{ Grãos kg/ } \\
\hline Testemunha & 2712 & 2625 & $2683 \mathrm{~b}$ & 2673 \\
\hline $\mathrm{KCl}$ & 2550 & 3224 & $3220 \mathrm{a}$ & 2998 \\
\hline $\mathrm{K}_{2} \mathrm{SO}_{4}$ & 2923 & 3074 & $3087 \mathrm{ab}$ & 3028 \\
\hline K-Mag & 2999 & 3149 & $2875 \mathrm{ab}$ & 3008 \\
\hline Vinhaça & 2858 & 3193 & $3212 \mathrm{a}$ & 3088 \\
\hline Média & 2808 & 3053 & 3015 & 2959 \\
\hline $\mathrm{CV} \%$ & 24,0 & 19,0 & 7,9 & 19,4 \\
\hline \multicolumn{3}{|c|}{ DMS (Tukey 5\%) trat. } & 465 & \\
\hline
\end{tabular}

TABELA 7 - Efeito da aplicação de adubos potássicos nos teores de macronutrientes nas folhas de soja de três anos agrícolas no ensaio de Mococa.

\begin{tabular}{|c|c|c|c|c|c|c|c|c|c|c|c|c|c|c|c|}
\hline \multirow{2}{*}{$\begin{array}{l}\text { Trata- } \\
\text { mento }\end{array}$} & \multicolumn{5}{|c|}{$1980 / 81$} & \multicolumn{5}{|c|}{$1981 / 82$} & \multicolumn{5}{|c|}{$1982 / 83$} \\
\hline & $\mathbf{N}$ & $\mathbf{P}$ & $\mathbf{K}$ & $\mathrm{Ca}$ & $\mathbf{M g}$ & $\mathbf{N}$ & $\mathbf{P}$ & $\mathbf{K}$ & $\mathrm{Ca}$ & $\mathbf{M g}$ & $\mathbf{N}$ & $\mathbf{P}$ & $\mathbf{K}$ & $\mathrm{Ca}$ & $\mathbf{M g}$ \\
\hline Test. & 3,8 & 0,28 & 3,0 & 1,1 & 0,49 & 3,9 & 0,33 & 2,1 & 1,6 & 0,60 & 3,0 & 0,25 & 1,0 & 1,3 & 0,53 \\
\hline $\mathrm{KCl}$ & 4,1 & 0,28 & 3,1 & 0,9 & 0,43 & 3,7 & 0,34 & 2,6 & 1,4 & 0,53 & 3,2 & 0,27 & 1,8 & 1,3 & 0,45 \\
\hline $\mathrm{K}_{2} \mathrm{SO}_{4}$ & 4,0 & 0,28 & 3,0 & 0,9 & 0,45 & 4,1 & 0,32 & 2,5 & 1,6 & 0,56 & 3,1 & 0,24 & 1,3 & 1,3 & 0,49 \\
\hline K-Mag & 4,0 & 0,28 & 3,0 & 1,0 & 0,46 & 4,0 & 0,33 & 2,7 & 1,6 & 0,58 & 3,0 & 0,25 & 1,5 & 1,3 & 0,50 \\
\hline Vinh. & 4,6 & 0,28 & 2,8 & 1,0 & 0,44 & 4,1 & 0,34 & 2,8 & 1,5 & 0,58 & 3,1 & 0,24 & 1,5 & 1,3 & 0,47 \\
\hline
\end{tabular}

TABELA 8 - Resultados da análise química do solo após a colheita de soja no ensaio de Mococa.

\begin{tabular}{|c|c|c|c|c|c|c|}
\hline \multirow[b]{2}{*}{ Tratamentos } & \multicolumn{3}{|c|}{$1981 / 82$} & \multicolumn{3}{|c|}{$1982 / 83$} \\
\hline & $\mathbf{K}$ & $\mathrm{Ca}$ & $\mathbf{M g}$ & $\mathbf{K}$ & $\mathrm{Ca}$ & $\mathbf{M g}$ \\
\hline \multicolumn{7}{|c|}{ 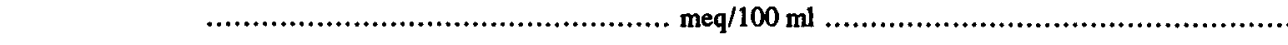 } \\
\hline Testemunha & 0,07 & 2,4 & 1,2 & 0,03 & 3,3 & 1,0 \\
\hline $\mathrm{KCl}$ & 0,12 & 2,6 & 1,4 & 0,06 & 3,3 & 1,0 \\
\hline $\mathrm{K}_{2} \mathrm{SO}_{4}$ & 0,09 & 2,3 & 1,2 & 0,05 & 3,5 & 1,0 \\
\hline K-Mag & 0,11 & 2,4 & 1,1 & 0,06 & 3,2 & 0,9 \\
\hline Vinhaça & 0,12 & 2,3 & 1,1 & 0,08 & 3,2 & 0,9 \\
\hline
\end{tabular}


TABELA 9 - Efeito da aplicação de diferentes adubos potássicos na produção de soja durante três anos agrícolas no ensaio de Ribeirão Preto.

\begin{tabular}{|c|c|c|c|c|}
\hline Tratamentos & $1980 / 81$ & $1981 / 82$ & $1982 / 83$ & Média \\
\hline & \multicolumn{4}{|c|}{ Grãos kg/ha } \\
\hline Testemunha & 1706 & 1888 & 1660 & 1751 \\
\hline $\mathrm{KCl}$ & 1934 & 1860 & 1847 & 1880 \\
\hline $\mathrm{K}_{2} \mathrm{SO}_{4}$ & 1805 & 1934 & 1988 & 1909 \\
\hline K-Mag & 2113 & 1913 & 1980 & 2002 \\
\hline Vinhaça & 2005 & 2176 & 2055 & 2079 \\
\hline Média & 1913 & 1954 & 1906 & 1924 \\
\hline $\mathrm{CV} \%$ & 22,0 & 16,0 & 11,3 & 16,6 \\
\hline DMS (Tukey 5\%) trat. & NS & NS & NS & NS \\
\hline
\end{tabular}

TABELA 10 - Efeito da aplicação de adubos potássicos nos teores de macronutrientes nas folhas de soja de três anos agrícolas no ensaio de Ribeirão Preto.

\begin{tabular}{|c|c|c|c|c|c|c|c|c|c|c|c|c|c|c|c|}
\hline \multirow{2}{*}{$\begin{array}{l}\text { Trata- } \\
\text { mento }\end{array}$} & \multicolumn{5}{|c|}{$1980 / 81$} & \multicolumn{5}{|c|}{$1981 / 82$} & \multicolumn{5}{|c|}{$1982 / 83$} \\
\hline & $\mathbf{N}$ & $\mathbf{P}$ & $\mathbf{K}$ & $\mathrm{Ca}$ & $\mathrm{Mg}$ & $\mathbf{N}$ & $\mathbf{P}$ & $\mathbf{K}$ & $\mathrm{Ca}$ & $\mathbf{M g}$ & $\mathbf{N}$ & P & $\mathbf{K}$ & $\mathrm{Ca}$ & $\mathrm{Mg}$ \\
\hline Test. & 4,0 & 0,26 & 2,0 & 1,8 & 0,68 & 5,4 & 0,22 & 1,7 & 1,7 & 0,66 & 3,9 & 0,33 & 1,4 & 2,4 & 0,60 \\
\hline $\mathrm{KCl}$ & 4,0 & 0,25 & 2,4 & 1,7 & 0,58 & 5,0 & 0,26 & 2,1 & 1,8 & 0,62 & 4,1 & 0,31 & 1,6 & 2,3 & 0,60 \\
\hline $\mathrm{K}_{2} \mathrm{SO}_{4}$ & 3,5 & 0,29 & 2,4 & 1,7 & 0,58 & 5,3 & 0,24 & 2,1 & 1,7 & 0,56 & 4,1 & 0,31 & 1,7 & 2,3 & 0,59 \\
\hline K-Mag & 4,0 & 0,27 & 2,4 & 1,8 & 0,63 & 5,4 & 0,23 & 2,3 & 1,7 & 0,57 & 4,0 & 0,31 & 1,7 & 2,2 & 0,62 \\
\hline Vinh. & 3,8 & 0,29 & 2,5 & 1,7 & 0,56 & 5,3 & 0,22 & 2,3 & 1,6 & 0,55 & 4,5 & 0,31 & 1,7 & 2,3 & 0,57 \\
\hline
\end{tabular}

TABELA 11 - Resuitados da análise química do solo após a colheita de soja no ensaio de Ribeirão Preto.

\begin{tabular}{|c|c|c|c|c|c|c|c|c|c|}
\hline \multirow[b]{2}{*}{ Tratamentos } & \multicolumn{3}{|c|}{$1980 / 81$} & \multicolumn{3}{|c|}{$1981 / 82$} & \multicolumn{3}{|c|}{$1982 / 83$} \\
\hline & $\mathbf{K}$ & $\mathrm{Ca}$ & $\mathbf{M g}$ & K & $\mathrm{Ca}$ & $\mathrm{Mg}$ & $\mathrm{K}$ & $\mathrm{Ca}$ & $\mathrm{Mg}$ \\
\hline & \multicolumn{9}{|c|}{$\mathrm{meq} / 100 \mathrm{ml}}$. \\
\hline Testemunha & 0,07 & 2,4 & 0,7 & 0,05 & 2,1 & 0,7 & 0,02 & 5,3 & 0,3 \\
\hline $\mathrm{KCl}$ & 0,22 & 2,3 & 0,7 & 0,08 & 2,1 & 0,6 & 0,02 & 3,6 & 0,3 \\
\hline $\mathrm{K}_{2} \mathrm{SO}_{4}$ & 0,15 & 2,7 & 0,8 & 0,08 & 2,4 & 0,7 & 0,02 & 3,9 & 0,4 \\
\hline K-Mag & 0,14 & 2,5 & 0,9 & 0,08 & 2,3 & 0,7 & 0,02 & 3,2 & 0,3 \\
\hline Vinhaça & 0,13 & 2,5 & 0,8 & 0,13 & 2,2 & 0,7 & 0,07 & 3,2 & 0,3 \\
\hline
\end{tabular}

Sci. agric., Piracicaba, 51(1):82-89, jan./abr., 1994 
TABELA 12 - Efeito da aplicação de diferentes fontes de potássio na produção de soja durante dois anos agrícolas no ensaio de Paraguaçu Paulista.

\begin{tabular}{|c|c|c|c|}
\hline Tratamentos & $1980 / 81$ & $1981 / 82$ & Média \\
\hline & & los $\mathrm{kg} / \mathrm{ha}$ & ......... \\
\hline Testemunha & 814 & $1065 \mathrm{~b}$ & 939 a \\
\hline $\mathrm{KCl}$ & 1054 & $1758 \mathrm{a}$ & $1406 \mathrm{ab}$ \\
\hline $\mathrm{K}_{2} \mathrm{SO}_{4}$ & 1140 & $1722 a$ & 1431 a \\
\hline K-Mag & 1007 & 1697 a & $1352 \mathrm{ab}$ \\
\hline Vinhaça & 990 & $1622 \mathrm{a}$ & $1306 \mathrm{ab}$ \\
\hline Média & 1001 & 1573 & 1287 \\
\hline CV \% & 22,2 & 19,0 & 17,6 \\
\hline DMS (Tukey 5\%) trat. & & 638 & 481 \\
\hline
\end{tabular}

TABELA 13 - Efeito da aplicação de adubos potássicos nos teores de macronutrientes nas folhas de soja de dois anos agrícolas no ensaio de Paraguaçu Paulista.

\begin{tabular}{|c|c|c|c|c|c|c|c|c|c|c|}
\hline \multirow[b]{2}{*}{ Tratamento } & \multicolumn{5}{|c|}{$1980 / 81$} & \multicolumn{5}{|c|}{$1981 / 82$} \\
\hline & $\mathrm{N}$ & $\mathbf{P}$ & $\mathrm{K}$ & $\mathrm{Ca}$ & $\mathrm{Mg}$ & $\mathrm{N}$ & $\mathbf{P}$ & $\mathbf{K}$ & $\mathrm{Ca}$ & $\mathrm{Mg}$ \\
\hline & & & & & & $\%$ & & & .... & $\ldots .$. \\
\hline Testesmunha & 4,7 & 0,39 & 2,1 & 1,2 & 0,64 & 3,0 & 0,29 & 0,74 & 2,0 & 0,75 \\
\hline $\mathrm{KCl}$ & 3,7 & 0,36 & 2,8 & 1,0 & 0,49 & 2,9 & 0,29 & 1,53 & 1,6 & 0,46 \\
\hline $\mathrm{K}_{2} \mathrm{SO}_{4}$ & 4,5 & 0,35 & 2,2 & 1,3 & 0,61 & 3,0 & 0,27 & 1,50 & 1,8 & 0,59 \\
\hline K-Mag & 4,2 & 0,38 & 2,9 & 0,9 & 0,55 & 3,1 & 0,29 & 1,50 & 1,5 & 0,58 \\
\hline Vinhaça & 4,4 & 0,33 & 2,2 & 1,1 & 0,59 & 3,2 & 0,28 & 1,65 & 1,9 & 0,63 \\
\hline
\end{tabular}

TABELA 14 - Resultados da análise química do solo após a colheita de soja no ensaio de Paraguaçu Paulista.

\begin{tabular}{|c|c|c|c|c|c|c|}
\hline \multirow[b]{2}{*}{ Tratamentos } & \multicolumn{3}{|c|}{$1980 / 81$} & \multicolumn{3}{|c|}{$1981 / 82$} \\
\hline & $\mathbf{K}$ & $\mathrm{Ca}$ & $\mathrm{Mg}$ & $\mathbf{K}$ & $\mathrm{Ca}$ & $\mathbf{M g}$ \\
\hline & & & $\ldots n$ & $\mathrm{ml} \mathrm{....}$ & ........ & $\ldots .$. \\
\hline Testemunha & 0,05 & 1,0 & 0,5 & 0,03 & 0,4 & 0,2 \\
\hline $\mathrm{KCl}$ & 0,11 & 1,0 & 0,2 & 0,03 & 0,4 & 0,2 \\
\hline $\mathrm{K}_{2} \mathrm{SO}_{4}$ & 0,07 & 1,1 & 0,3 & 0,04 & 0,4 & 0,1 \\
\hline K-Mag & 0,14 & 1,1 & 0,3 & 0,03 & 0,3 & 0,1 \\
\hline Vinhaça & 0,06 & 1,0 & 0,3 & 0,03 & 0,3 & 0,1 \\
\hline
\end{tabular}


estavam bem abaixo do nivel adequado, enquanto nos outros tratamentos estão ligeiramente abaixo do nível adequado, com exceção do tratamento com $\mathrm{KCl}$ (TABELA 7).

Os teores de potássio no solo também estão baixos, principalmente na testemunha (TABELA 8). Nos três anos agrícolas do ensaio de Ribeirão Preto não houve efeito de adubos potássicos mesmo quando comparados com a testemunha (TABELA 9). Novamente evidenciou-se que o cultivar IAC-9 teve alta capacidade de extrair $\mathrm{K}$ e $\mathrm{Mg}$ do solo que apresentou baixa disponibilidade (TABELA 11) comprovado pelos dados de TABELA 10.

No ensaio de Paraguaçu Paulista as produtividades no primeiro ano são baixas podendo ser consideradas normais para o cultivo inicial de soja em solo de cerrado de baixa fertilidade natural (TABELA 12). Os teores de potássio nas folhas indicam que todos os tratamentos proporcionaram níveis adequados, confirmando os dados de MASCARENHAS et al. (1976). A ausência de resposta pode ser devido a restos de matéria orgânica que se decompõem mais rapidamente com a calagem, liberando nutrientes às plantas. No segundo ano (1981/82), observaram-se sintomas de deficiência de potássio nas folhas de soja da testemunha, evidenciados pela clorose, que não foram verificados nos demais tratamentos. Isto refletiu nos teores de potássio nas folhas e na produtividade (TABELAS 12 e 13). No segundo ano os teores de $\mathrm{K}, \mathrm{Ca}$ e $\mathrm{Mg}$ no solo estavam muito baixos pelo fato do mesmo ser muito arenoso o que aumentava o potencial de lixiviação daqueles nutrientes. Os dados de $1982 / 83$ não são apresentados devido ao veranico que prejudicou o rendimento.

Nos ensaios de Orlândia, Mococa, Ribeirão Preto e Paraguaçu Paulista foram aplicadas nos três anos respectivamente $446,402,473$ e $454 \mathrm{~kg} / \mathrm{ha}$ de $\mathrm{K}_{2} \mathrm{O}$ na forma de vinhaça que são doses superiores em até três vezes do adubo mineral aplicado. Apesar dessas dosagens, tanto no solo quanto nas folhas de soja foram observadas diminuições de potássio com os cultivos.

CAMARGO et al. (1987) mostraram que há perdas dos cátions $\mathrm{K}, \mathrm{Ca}$ e $\mathbf{M g}$ em períodos chuvosos em vinhaça aplicada, podendo ser superiores a $60 \%$.

De modo geral o potássio aplicado somente aumenta a produtividade, quando os teores no solo são muito baixos, conforme já apontado por MASCARENHAS et al. (1971). O efeito de potássio é mais indireto, como melhorar a qualidade fisiologica e sanitária das sementes, e aumentar o teor de óleo.

\section{CONCLUSÕES}

1. Houve diminuição gradativa de potássio no solo como também nas folhas de soja com os cultivos.

2. O cultivar de soja IAC-9 apresentou alta capacidade extratora tanto do $\mathrm{K}$ como de $\mathrm{Ca}$ e $\mathrm{Mg}$.

3. Houve respostas positivas ao uso de potássio em Paraguaçu Paulista no segundo ano e em Mococa no terceiro ano, não havendo diferenças entre as fontes.

\section{REFERENCIAS BIBLIOGRÁFICAS}

BATAGLIA, O.C.; FURLANI, M.A.C.; TELXEIRA, J.P.F.; FURLANI, P.R.; GALLO, J.R. Metodos de andilises de plantas. Campinas: Instituto Agronômico, 1983. 48p. (Boletim Técnico, 78).

CAMARGO, O.C. de; VALADARES, J.M.A.S.; BERTON, R.S.; TEOFILO SOBRINHO, J.; MENK, J.R.F. Alteração das caracteríticas químicas de um Latossolo Vermelho-Escuro distrofico pela aplicação de vinhaça. Campinas: Instituto Agronômico, 1987. 23p. (Boletim Científico, 9).

MASCARENHAS, H.A.A.; DEMATTE, J.D.; MIYASAKA, S.; IGUE, T. Estudos preliminares sobre a adubação economica da soja (Glycine max (L.) Merrill na regiāo de Alta Mogiana, em Latosol Roxo e Latosol Escuro fase arenosa. Campinas: Instituto Agronômico, 1971. 7p. (Projeto BNDE/ ANDA/CIA, 3).

MASCARENHAS, H.A.A.; GALLO, J.R.; RAIJ, B.; IGUE, T.; BATAGLIA, O.C. Efeito da calagem nas características químicas do solo e na nutrição de soja em Latossolo Roxo distrófico de cerrado. Bragantia, Campinas, v.35, p.273-278, 1976.

OHLROGGE, A.J.; KAMPRATH, E.J. Fertilizer use in soybeans. In: DINAUR, R.C. Ed. Changing patterns in fertilizer use. Madison: Soil Science Society of America, 1968. p.275-295.

PARKER, M.B.; GASCHO, G.J.; GAINES, T.P. Chloride toxicity of soybeans grown on Atlantic Coast Flatwoods soils. Agronomy Journal, Madison, v.75, n.3, p.439-443, 1983.

RAJ, B.; QUAGGIO, J.A. Métodos de analise de solo para fins de fertilidade. Campinas: Instituto Agronômico, 1983. 31p. (Boletim Técnico, 81).

SEARLS, J.P. Potash: mineral comodity profiles. Washington: U.S. Dept. of the Interior, p.28. 1983.

Enviado para publicação em 23.06 .93

Aceito para publicaçĩo em 06.10 .93 